Case report

\title{
Cervical lymph node dissemination of a multirecurrent supratentorial ependymoma
}

\author{
Marie-Amelyne Le Rouzic ${ }^{1,{ }^{*}}$, Julie Valduga ${ }^{1}$, Fanny Fouyssac ${ }^{1}$, Emmanuelle \\ Schmitt ${ }^{2}$, Ludovic Mansuy ${ }^{1}$, Laurent Coffinet $^{3}$, Valérie Bernier ${ }^{4}$ \\ ${ }^{1}$ Department of Pediatric Hematology and Oncology, Children's University Hospital, Nancy, France, \\ ${ }^{2}$ Department of Pediatric Neuroradiology, University Hospital, Nancy, France, ${ }^{3}$ Department of \\ Pediatric Otorhinolaryngology, Children's University Hospital, Nancy, France, ${ }^{4}$ Department of \\ Radiotherapy, Institut de Cancérologie en Lorraine, Nancy, France
}

\begin{abstract}
Ependymomas are rare central nervous system tumors. Half the patients experience recurrence and total resection is of major importance to obtain the most chance of cure. Thus, some patients undergo several repeated resections which may occasionally be at the origin of dissemination along the neuraxis and exceptionally to extraneural sites. We report the case of a boy who presented a multiple recurrence of his supratentorial ependymoma, first at initial site and then with cervical lymph nodes metastases, highlighting the fact that multiple surgical resections may confer an added risk for hematogeneous and lymphatic dissemination. The impact of adjuvant therapies on this risk is discussed.
\end{abstract}

Keywords: ependymoma; child; surgical dissemination; extraneural metastases

\section{Introduction}

Ependymoma is the third most common pediatric primary brain tumor and accounts for about $10 \%$ of pediatric central nervous system (CNS) neoplasms [1]. They develop in all age groups. However, their incidence depends on histological variant, molecular group and location. About $30 \%$ of pediatric ependymomas are diagnosed in children younger than three years old. These tumors can arise anywhere in the neuraxis but locate predominantly in the posterior fossa, in about two thirds of the cases.

Received: January 2018; Accepted after review: May 2018; Published: June 2018.

${ }^{*}$ Corresponding author: Marie-Amelyne Le Rouzic, Department of Pediatric Hematology and Oncology, Children's University Hospital, 11 Allée du Morvan, 54511 Vandoeuvre, France.

Email: m.lerouzic@chru-nancy.fr
The supratentorial tumors occur more frequently in older children and adolescents. The 2007 World Health Organization (WHO) classification of primary brain tumors distinguished four types of ependymal tumors based on their histological appearance: subependymoma (WHO grade I), myxopapillary ependymoma (WHO grade I), ependymoma with cellular, papillary and clear cell variants (WHO grade II) and anaplastic ependymoma (WHO grade III) [2]. Surgery is the mainstay of therapy associated with local radiation therapy. The role of chemotherapy is not proven. Moreover, each treatment can provide severe late effects, particularly radiotherapy, almost in the young age group of patients and in the case of supratentorial tumor location. While surgery is of major importance for the outcome, iterative resections could lead to intra- and extra-neural metastases as observed in our case and in the very few cases reported in the literature. 


\section{Case report}

A 32-month-old boy presented with clinical features of raised intracranial pressure for the past two weeks. The brain MRI showed a right frontal lobe cystic and solid lesion $(7 \times 7 \times 7 \mathrm{~cm})$ (Figure 1). A complete macroscopic resection of the tumor was performed. Histology revealed a clear cell ependymoma according to the $2007 \mathrm{WHO}$ brain tumor classification used at that time. The MIB 1 (Ki-67) proliferative index was estimated to be around $10 \%$. Due to the complete resection and the young age, only a close clinical and radiological surveillance was planned.

Twenty-one months later, MRI showed a relapse at the initial site and in the right temporal lobe. A second complete macroscopic resection of both lesions was performed. The neuropathological analysis confirmed the diagnosis of clear cell ependymoma. Considering the parents' refusal of radiation therapy and the absence of therapeutic target for chemotherapy, no treatment was undertaken and the child was regularly followed-up in consultation.

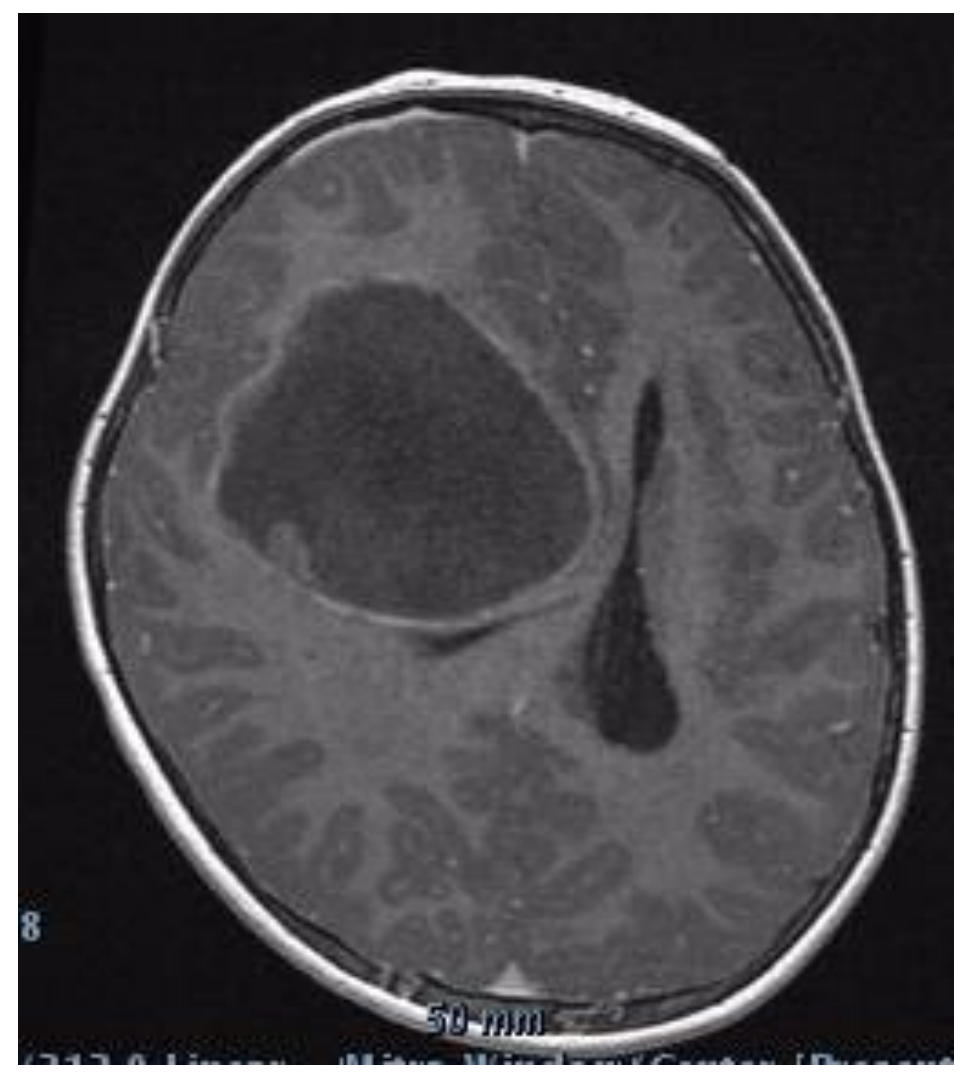

Fig. 1. Axial section of brain MRI (T1-weighted) showing right frontal ependymoma

Sixteen months later, MRI showed another focal tumor recurrence at the initial site. A third complete macroscopic resection was performed and proton therapy was then planned. However, clinical examination prior to radiotherapy found the occurrence of a painless pre-auricular mass (Figure 2). A biopsy was performed and histological analysis concluded to a lymph node metastasis of the ependymomas. Body CT scan, PET scan and bone marrow biopsies ruled out other metastases. Although the efficacy of chemotherapy is not yet proven in ependymoma primary brain tumors, partly due to the poor blood-brain barrier drug penetration, we considered that ENM were not subject to this same restriction and a polychemotherapy combining vincristine, etoposide and cyclophosphamide (VEC protocol), reported to display some responses [3], was initiated. After two courses, the tumor size had reduced by $27 \%$. A third course was ineffective. Surgery with parotidectomy, right cervical lymph node curage and excision of the lower part of the scalp scar was performed. Histological analysis of the parotid 
gland, all the lymph nodes, except one, and the skin yielded normal results. The primitive and metastatic areas were irradiated at the dose of 59.4 Gy. Unfortunately, the child relapsed 9 months later in the brain. The parents refused any other treatment, leading to death due to tumor progression 7.8 years after the diagnosis.

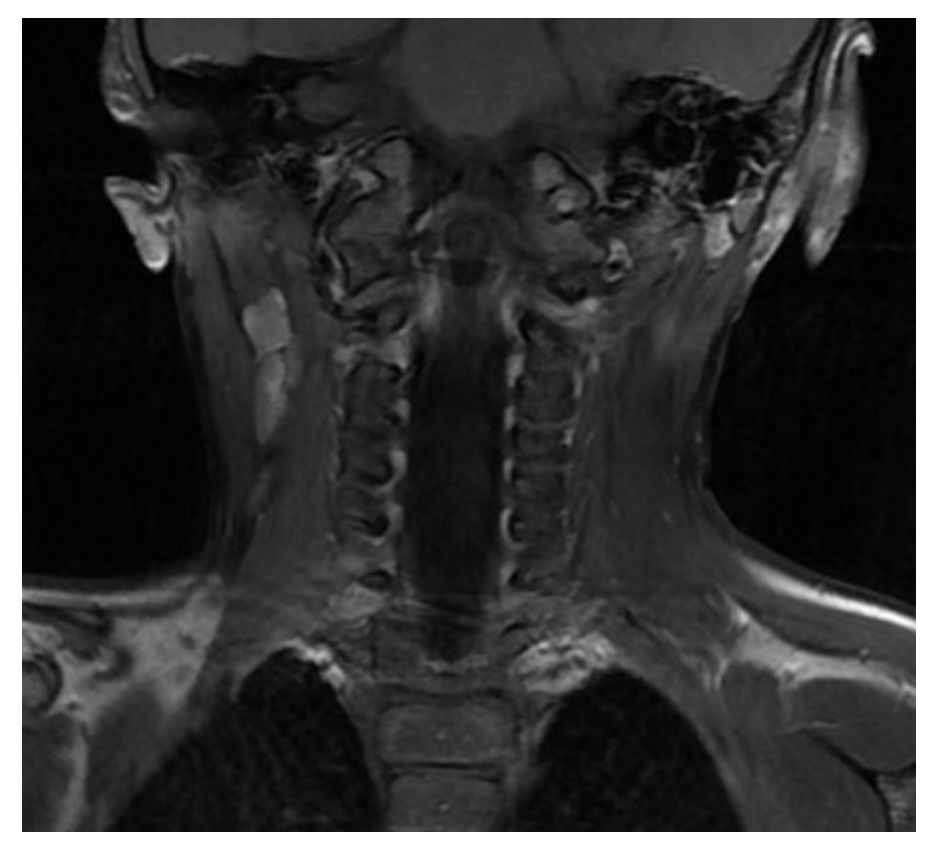

Fig. 2. MRI coronal section of cervical region with multiple lymph nodes

\section{Discussions}

Ependymoma patients remain a great challenge due to the lack of chemosensitivity (contrary to other malignant primary brain tumors in children like medulloblastomas and germ cell tumors) and the need for high radiation doses which are particularly deleterious in young patients. Thus, total surgical removal is of major importance. It has been reported that a gross total resection could be obtained in more than $80 \%$ of the cases through repeated resections [4]. Standard therapy includes maximal safe surgical resection followed by adjuvant therapy including irradiation in most cases, with or often without chemotherapy. Two factors have been consistently described as being prognostic across several series in the literature: age and extent of surgical excision, but total resection is associated with a better overall survival (OS) rate independently of age [4]. Overall survival rates range from $24 \%$ to $75 \%$ at 5 years, and at least, one third to half the patients experience relapse. The 10-year OS rate is $64 \%$ in pediatric patients [1].
Radiotherapy aims at eradicating tumor cells within the cerebrospinal fluid (CSF), thus preventing meningeal metastasis and possible neoplastic re-seeding, and could be recommended in case of incomplete resection, particularly for posterior fossa locations. However, the high frequency of radio-induced neurocognitive and endocrine sequelae, particularly in the youngest children and in case of supratentorial location, contributes to treatment reluctance as observed thrice in our case. Chemotherapy has been used in ependymomas, especially in very young children, with the aim of avoiding radiotherapy, but there is only little evidence that this therapeutic approach improves overall survival [5].

Considering all brain tumors, metastatic disseminations are rare and located within the CNS in almost all cases, extraneural metastases (ENM) having been reported in only very few cases. In pediatric patients, the most common CNS tumor types leading to ENM were: medulloblastoma (56.3\%), germinoma (9.8\%), glioblastoma $(6.9 \%)$, ependymoma $(3.7 \%)$ and pilocytic 
astrocytoma (2.9\%) [6]. Metastatic sites varied with tumor type but approximately one quarter of metastases occurred with the use of a shunt [6].

Proliferation and dissemination kinetics of ependymomas remain unpredictable. Intracranial ependymomas may spread by local infiltration in the surrounding brain or by dissemination through the CSF [7]. EN metastases mostly concern lymph nodes and visceral organs such as the lungs [7, 8]. Multiple surgical procedures appear to be the most significant risk factor for EN spread [9, 10]. In our patient who underwent three tumor resections, surgery may have added a risk for lymph node metastasis. The cervical site of his ENM suggests spread via haematogenous and lymphatic pathways. Only 12 pediatric ependymoma ENM have been well described in the literature $[8,11-17]$. In these series, the median age was 6 years. Among the 7 patients for whom the primary tumor location was reported, they were as follows: temporal (2), spinal (1), plexus choroid (1), occipital (1), frontal (1), parietal (1) [11-17]. Neuropathology was described in 6 cases and yielded results in favor of an anaplastic type in 5 cases and a grade $I$ in 1 case [11-13, 15-17]. Repeated resections were performed (range: 2-4) in 3 of 6 reported cases [11-16]. ENM were located in lymph nodes (4 cases), liver (2 cases), scalp ( 2 cases), bones (2 cases), lungs ( 1 case) [1117]. The predominance of lymph node metastasis seems to be characteristic of ependymoma tumors contrary to medulloblastomas or germinomas for which bones and visceral organs are mostly involved [6].

The presence of metastases is associated with a very poor prognosis, leading to death in pediatric series [18]. Interestingly, no significant difference in the incidence of spinal metastases when analyzed by grade and extent of radiotherapy field was observed in the series of Vanuytsel et al. [18]. Indeed, three out of 36 patients (i.e. $8 \%$ ) who received spinal radiotherapy developed spinal metastases, compared to one out of 10 patients (i.e. 10\%) without spinal radiotherapy [18]. This questioned the role of radiotherapy in preventing tumor cell dissemination. Although a minor response on the supratentorial location was observed using the VEC chemotherapy regimen, it did not suffice to avoid metastatic dissemination (supposing some degree of chemosensitivity).

As in our case, none of the 12 pediatric ependymoma ENM that have been well described in the literature was classified according to the WHO 2016 classification which has incorporated modifications, by deleting the cellular variant of these tumors and integrating the RELA fusion-positive subtype as a specific entity [19]. Clear cell ependymoma was one of the 4 main histological subtypes of ependymomas defined by this classification. The clear cell subtype of ependymoma was found to have a predilection for extraneural dissemination, mainly to cervical lymph nodes [20].

In the literature, ENM were observed with a delay of 2 to 6 years from diagnosis (median: 3 years) in accordance with the 3year delay observed in our case [11-13, 1517]. Long-term follow-up is thus recommended for patients harboring ependymomas due to the tendency of these tumors towards multiple and possibly late recurrences despite radical surgery and radiation therapy [7].

\section{Conclusions}

It is very difficult to draw some recommendations based on our own case and on the few other published cases because of the challenge to cure these young children harboring intracranial ependymomas and avoid severe treatment-induced sequelae. Although total resection is mandatory to obtain cure, we can only insist on the fact that clinicians must be aware of the necessity to assess ENM, especially in case of multiple resections.

\section{Competing interests}

The authors declare that they have no competing interests. 


\section{References}

1. Ostrom QT, de Blank PM, Kruchko C, et al. Alex's Lemonade Stand Foundation infant and childhood primary brain and central nervous system tumors diagnosed in the United States in 2007-2011. Neuro Oncol 2015; 16(10):1-36.

2. Louis DN, Ohgaki $H$, Wiestler OD, et al. The 2007 WHO classification of tumors of the central nervous system. Acta Neuropathol 2007; 114(2):97-109.

3. Massimino M, Gandola L, Giangaspero F, et al. Hyperfractionated radiotherapy and chemotherapy for childhood ependymoma: final results of the first prospective AIEOP (Associazione Italiana di Ematologia-Oncologia Pediatrica) study. Int J Radiat Oncol Biol Phys 2004; 58(5):1336-1345.

4. Merchant TE, Li C, Xiong X, Kun LE, Boop FA, Sanford RA. Conformal radiotherapy after surgery for pediatric ependymoma: a prospective study. Lancet Oncol 2009; 10(3):258-266.

5. Khatua S, Ramaswamy V, Bouffet E. Current therapy and the evolving molecular landscape of pediatric ependymoma. Eur J Cancer 2017; 70:34-41.

6. Rickert $\mathrm{CH}$. Extraneural metastases of pediatric brain tumors. Acta Neuropathol 2003; 105(4):309- 327.

7. Bademci G, Tun K, Erden E, Evliyaoglu C, Unlu A. Late dissemination of ependymoma: case report. Neurocirugia (Astur) 2007; 18(4):333336.

8. Newton HB, Henson J, Walker RW. Extraneural metastases in ependymoma. $J$ Neurooncol 1992; 14(2):135-142.

9. Hoffman HJ, Duffner PK. Extraneural metastases of central nervous system tumors. Cancer 1985; 56(7 Suppl):1778-1782.

10. Duffner PK, Cohen ME. Extraneural metastases in childhood brain tumors. Ann Neurol 1981; 10:261-265.

11. Chao M, Packer RJ, Myseros JS, Rood BR. Isolated extracranial recurrence of anaplastic ependymoma. Pediatr Blood Cancer 2011; 56(2):317-318.

12. Dunst $J$, Klinger $M$, Thierauf $P$. Ependymoma with cervical lymph node metastases. Klin Padiatr 1987; 199(1):19-21.

13. Kinoshita M, Izumoto $S$, Kagawa N, Hashimoto $\mathrm{N}$, Maruno M, Yoshimine T. Long-term control of recurrent anaplastic ependymoma with extracranial metastasis: importance of multiple surgery and stereotactic radiosurgery procedures-case report. Neurol Med Chir 2004; 44:669-673.

14. Varan A, Sari N, Akalan N, et al. Extraneural metastasis in intracranial tumors in children: the experience of a single center. $J$ Neurooncol 2006; 79(2):187-190.

15. Kumar $P$, Rastogi $N$, Jain $M$, Chhabra $P$. Extraneural metastases in anaplastic ependymoma. J Cancer Res Ther 2007; 3(2):102-104.

16. Hussain $M$, Mallucci $C$, Abernethy $L$, Godhamgaonkar V, Thorp N, Pizer B. Anaplastic ependymoma with sclerotic bone metastases. Pediatr Blood Cancer 2010; 55(6):1204-1206.

17. Fischer C, Haque SS, Huse JT, et al. Extraneural ependymoma: distant bone, lung, liver, and lymph node metastases following bevacizumab. Pediatr Blood Cancer 2013; 60(1):143-145.

18. Vanuytsel LJ, Bessell EM, Ashley SE, Bloom HJ, Brada M. Intracranial ependymoma: longterm results of a policy of surgery and radiotherapy. Int J Radiat Oncol Biol Phys 1992; 23(2):313-319.

19. Fouladi M, Helton K, Dalton J, et al. Clear cell ependymoma: a clinicopathologic and radiographic analysis of 10 patients. Cancer 2003; 98(10):2232-2244.

20. Louis DN, Perry A, Reifenberger G, et al. The 2016 World Health Organization classification of tumors of the central nervous system: a summary. Acta Neuropathol 2016; 131(6):803820. 